\title{
O: Oral presentations
}

\section{A:20 HELICOBACTER PYLORI RESISTANCE TO MACROLIDES. CONFIRMATION OF POINT MUTATION AND DETECTION BY PCR-RFLP}

A. Occhialini ${ }^{1}$, M. Urdaci ${ }^{2}$, F. Doucet-Populaire ${ }^{3}$, H. Lamouliatte ${ }^{4}$, C. Bébéar ${ }^{1}$, F. Mégraud ${ }^{1}{ }^{1}$ Hôpital Pellegrin, Paris; ${ }^{2}$ University of Bordeaux I, Bordeaux; ${ }^{3}$ CHU Pitie Salpétrière, Paris; Hôpital Mignot, Le Chesnay; ${ }^{4}$ Hôpital St. André, Bordeaux, France

Resistance of Helicobacter pylori to macrolides is a major cause of treatment failure of eradication therapies. A princeps work based on U.S. strains showed that this resistance was due to a point mutation on the gene coding for the 23S ribosomal RNA of $H$. pylori. Our goal was to extend this work using European strains, to test the consequence of this mutation on erythromycin binding to $H$. pylori ribosomes, and to find a quick method to detect this mutation. The reference strain CIP 101260 and 7 pairs of $H$. pylori strains were used, the parent strain being susceptible to macrolides, and a second strain having acquired resistance in vivo during clarithromycin treatment. The identity of the strains was confirmed by RAPD using 2 primers. The MIC values were measured by $E$ test and agar dilution method. Amplification was carried out on the peptidyl transferase loop region of the gene coding for the $23 \mathrm{~S}$ rRNA using appropriate primers in order to confirm the presence of the mutations 2058, 2059 and 2611 previously described. The amplified products were sequenced. Erythromycin binding was tested on purified ribosomes isolated from one pair of strains using ${ }^{14} \mathrm{C}$ labelled erythromycin.

A point mutation was found on resistant strains at position 2058 ( 3 cases) and 2059 (4 cases) but never on the opposite DNA fragment of domain $\mathrm{V}$ of the gene. The mutation was $\mathrm{A} \rightarrow \mathrm{G}$ in all except one case $(\mathrm{A} \rightarrow \mathrm{C})$ in position 2058. Erythromycin binding increased in a dose dependent manner for the susceptible strain but not for the resistant one. Using $B s a l$ and $B b s I$ restriction enzymes on the amplified products, we could confirm the mutations in positions 2059 and 2058, respectively.

In conclusion, the same mutations as previously found were present in European strains. This mutation correlates with a decreased uptake of macrolide by ribosomes. Finally the mutation could be detected without sequencing by performing PCR-RFLP using appropriate restriction enzymes.

\section{C:06 DOES HELICOBACTER PYLORI INFECTION HAVE A ROLE IN PERNICIOUS ANEMIA?}

G.I. Pérez-Pérez, R. Carmel, M.J. Blaser. Vanderbilt University Department of Veterans Affairs Medical Center, Nashville TN; University of Southern California Los Angeles CA

Previous studies indicate that patients with pernicious anemia (PA) are infected with $H$. pylori (Hp) less often than are age-matched controls. However, Hp infection may be present prior to the development of PA and gradually become absent due to the development of the associated type A atrophic gastritis. To determine whether evidence of Hp infection may disappear during the course of the illness, we studied 47 PA patients (mean age $58.2 \mathrm{y}$ and $36.2 \%$ male) prospectively. The diagnosis of PA was established by either: i) abnormal Schilling test corrected by intrinsic factor (IF); ii) lack of IF in stimulated gastric juice or iii) the combination of low $B_{12}$ levels and serum anti-IF antibodies. Sera were available for a mean of $5.9 \pm 0.5$ years and ELISAs were used to detect Hp-specific serum IgG or IgA. In total, 12 (25.5\%) patients showed evidence of Hp infection. Comparison of the $12 \mathrm{Hp}$-seropositive (Hp+) and $35 \mathrm{Hp}$ seronegative ( $\mathrm{Hp}-$ ) PA patients showed that the former group had significantly lower gastrin (mean $686 \mathrm{vs} 1293 \mathrm{pg} / \mathrm{ml}, \mathrm{p}=0.045$ ) and higher pepsinogen $\mathrm{A}$ levels (mean 27.1 vs. $11.6 \mathrm{pg} / \mathrm{ml}, \mathrm{p}=0.05$ ), factors consistent with less severe atrophic gastritis. The $35 \mathrm{Hp}$ - patients were followed for a total of 204 person-years; none showed seroconversion. The $12 \mathrm{Hp}+$ were followed for a total of 63 person-years, (mean 5.25 years); during this time, 4 (33\%) of the patients seroreverted. Thus, among patients with PA, the seroreversion rate was greater than $6 \%$ per year. Those who seroreverted were younger (52 vs. $69 \mathrm{y}, \mathrm{p}=0.06$ ) and had lower pepsinogen A levels ( 9 vs. $39, \mathrm{p}$ $=0.057$ ) than those remaining positive. These data are consistent with the hypothesis that $\mathrm{Hp}$-infection precedes at least some cases of PA and that the infection reverses with time; and presumably with progressive gastritis. This pattern may be especially associated with a subset of PA characterized by less severe gastrin and pepsinogen abnormalities in infected persons. The development of atrophic gastritis as part of PA leads to gradual elimination of the organism and failure to detect a past $\mathrm{Hp}$ infection. Prospective studies, in the pre-PA stage of gastritis are needed to clarify the putative role of $\mathrm{Hp}$ in causing PA.

\section{C:13 HELICOBACTER INFECTION AND THE RISK OF MYOCARDIAL INFARCTION}

A. Aromaa ${ }^{1}$, P. Knekt ${ }^{1}$, A. Reunanen ${ }^{1}$, H.I. Rautelin ${ }^{2}$, T.U. Kosunen ${ }^{2}$. ${ }^{1}$ National Public Health Institute and Social Insurance Institution, Helsinki, Finland; ${ }^{2}$ University of Helsinki, Helsinki, Finland

Certain infections including those caused by helicobacters, may increase the risk of cardiovascular diseases. Our purpose was to analyse the association of helicobacter infection with myocardial infarction in the general population.

Methods. A health examination survey was carried out in 12 Finnish cohorts during 1973-76 with a follow-up until the end of 1985. $3471 \mathrm{men}$, aged 45-64 years, participated in the baseline survey. During the follow-up a new fatal or non-fatal myocardial infarction was observed in 276 subjects who were free of cardiovascular disease at baseline and in 165 subjects who had cardiovascular disease at baseline. A nested case-control study based on these cases and two matched control subjects was performed. A total of 842 controls were matched for age, sex and municipality and cardiovascular disease at baseline. Serum samples taken at baseline were analyzed for helicobacter IgG and IgA antibodies.

Results. $79 \%$ of the subjects with cardiovascular disease at baseline and myocardial infarction during the follow-up had elevated titers of helicobacter IgG antibodies, versus $77 \%$ in their control subjects, which resulted in a relative risk (RR) of 1.16; $95 \%$ confidence interval (CI) $0.73-1.86$. Of those who initially did not have cardiovascular disease but had first myocardial infarction during the follow-up, $83 \%$ had IgG antibodies at the baseline, versus $78 \%$ in the control subjects; RR 1.37 , (95\% CI 0.94-2.00) Overall IgG positivity of the 441 subjects with myocardial infarction was $82 \%$, versus $78 \%$ in the control subjects, RR 1.29 (95\% CI 0.96-1.73). The respective figures for IgA antibodies were $68 \%$ and $67 \%$, RR 1.05 (95\% CI 0.82-1.34).

Conclusion. The present data indicate a possible although statistically nonsignificant association between the risk of myocardial infarction and helicobacter infection as measured by IgG positivity. However, this possible risk seems to be small.

\section{A:07 AN INTERNATIONAL ASSOCIATION BETWEEN PREVALENCE OF INFECTION WITH CagA POSITIVE STRAINS OF $H$. PYLORI AND MORTALITY FROM GASTRIC CANCER}

P.M. Webb ${ }^{1}$, D. Forman, D. Newell ${ }^{2}$, A. Covacci ${ }^{3}$, J.E. Crabtree and the Eurogast Study Group. University of Leeds \& St. James's Hospital, Leeds, UK; ${ }^{1}$ University of Queensland, Brisbane, Australia; ${ }^{2}$ Central Vet Labs, Weybridge, UK; ${ }^{3}$ IRIS, Siena, Italy

Purpose: There has been considerable recent interest in whether individuals infected strains of $H$. pylori, expressing the cagA gene product, have an increased risk of gastric cancer in comparison with those infected with cagA negative strains. This question has been investigated in the Eurogast study in which we have previously demonstrated an international correlation between gastric cancer mortality rates and $H$. pylori infection prevalence.

Methods: A total of 2839 serum samples collected in 1990-2 from approximately 50 men and 50 women in the age groups 25-34 and 55-64 years from each of the 17 Eurogast study centres, were analysed in a single laboratory for the presence of IgG antibodies to the CagA protein by an ELISA using as antigen a purified recombinant fragment of CagA. Regression analyses were then then carried out in which the prevalence of CagA seropositivity was modelled on the cumulative (0-74 yrs) gastric cancer mortality rate for each population in the mid-1980's.

Results: There was a statistically significant association $(p=0.01)$ between the prevalence of individuals with CagA seropositivity in each population and the mortality rate from gastric cancer. The regression coefficient (2.76) was higher than that for the effect of $H$. pylori seropositivity (1.72) although the latter was of similar significance $(p=0.002)$. Based on these data, the relative risk of gastric cancer associated with CagA positive $H$. pylori infection would be 15.8 in comparison with a risk of 5.6 associated with $H$. pylori per se. 
Conclusions: These results provide evidence to suggest that the worldwide variation in gastric cancer mortality may be associated more strongly with the variation in infection with CagA positive strains than with CagA negative strains of $H$. pylori.

\section{B:05 THE ${ }^{13} \mathrm{C}$-UREA BREATH TEST FOR THE DIAGNOSIS OF HELICOBACTER PYLORI INFECTION IN CHILDREN}

The Bologna ${ }^{13}$ C-Urea Breath Test User Group, F. Bazzoli, L. Cecchini ${ }^{1}$, L. Corvaglia, M. Dall'Antonia ${ }^{6}$, M. Dalla Libera ${ }^{2}$, C. De Giacomo ${ }^{4}$, S. Fossi, P. Garisio ${ }^{5}$, L. Gobbio Casali ${ }^{3}$, S. Gullini ${ }^{2}$, R. Lazzari, G. Leggeri $^{3}$, P. Lerro ${ }^{5}$, F. Lizzoli ${ }^{4}$, G. Mandrioli ${ }^{2}$, M. Marani ${ }^{1}$, P. Martelli ${ }^{5}$, A. Miano ${ }^{1}$, C. Mwangemi, G. Oderda ${ }^{5}$, A. Pasetti, P. Pazzi ${ }^{2}$, P. Pozzato, L. Ricciardiello, E. Roda, P. Simoni, S. Sottili, G. Torre ${ }^{6}$, L. Urso ${ }^{6}$, R.M. Zagari. Bologna; ${ }^{1}$ Cesena; ${ }^{2}$ Ferrara; $;{ }^{3}$ Mantova; ${ }^{4}$ Pavia; ${ }^{5}$ Torino; ${ }^{6}$ Trieste, Italy

Background. The ${ }^{13} \mathrm{C}$-Urea Breath Test $\left({ }^{13} \mathrm{C}\right.$-UBT $)$ is a simple non-invasive highly accurate test for the detection of Helicobacter $(\mathrm{H}$.) pylori infection in adults. Although the use of ${ }^{13} \mathrm{C}$-labelled urea renders this test absolutely safe and thus undoubtedly suitable for the detection of $\mathrm{H}$. pylori infection in children, as yet a standardized ${ }^{13} \mathrm{C}$-UBT protocol for children has not been formulated. In particular we have no information on the three fundamental components of the ${ }^{13} \mathrm{C}$-UBT: the number of and time intervals for breath sample collection, the appropriate test meals to delay gastric emptying and doses of ${ }^{13} \mathrm{C}$-Urea. Aim. The aim of our study was to evaluate the accuracy of the ${ }^{13} \mathrm{C}$-UBT in children using different types of test meal, doses of ${ }^{13} \mathrm{C}$-Urea and breath sampling intervals. Methods. 98 children, recruited in our study (51 males, 47 females; age (yrs) range 2-16, mean \pm SE: 10.1 \pm 0.3 ; body surface area $\left(\mathrm{m}^{2}\right)$ range $0.5-1.7$, mean $\left.\pm \mathrm{SE}: 1.2 \pm 0.03\right)$ underwent routine upper GI endoscopy. 3 antral and 2 corpus-fundus biopsy specimens were taken for histological evaluation for the presence of $\mathrm{H}$. pylori infection (Haematoxylin/Eosin; GIEMSA) and the quick Urease-test was performed. The ${ }^{13} \mathrm{C}$-UBT was performed in each child after undergoing endoscopy, and was then repeated within three days modifying the test meal or the dose of the ${ }^{13} \mathrm{C}$-Urea. 62 children were given a fatty test meal, Pulmocare (Abbott) $100 \mathrm{ml}$, and two different doses of ${ }^{13} \mathrm{C}$-Urea, 100 and $50 \mathrm{mg}$ respectively. 36 children were given the same dose of ${ }^{13} \mathrm{C}$-Urea, 50 $\mathrm{mg}$, but two different types of test meal, Pumocare $100 \mathrm{ml}$ and $10 \mathrm{~g}$ at $10 \%$ of Polycose (polymer of glucose) respectively. Breath samples were collected every 10 minutes for 60 minutes and analyzed by an Automated Breath ${ }^{13} \mathrm{C}$ Analyzer (ABCA Europa Scientific). The "gold standard" for the detection of $\mathrm{H}$. pylori infection was defined as a concordant result on histology and quick urease-test. The cut-off value was calculated taking the mean of $\mathrm{H}$. pylori - ve subjects $\pm 3 \mathrm{SD}$ and using a ROC curve. Results. According to the "gold standard" 48 children were considered H. pylori +ve and $44 \mathrm{H}$. pylori -ve.

\begin{tabular}{|c|c|c|c|c|c|c|}
\hline & T10 & $\mathrm{T} 20$ & T30 & $\mathrm{T} 40$ & T50 & T60 \\
\hline $\begin{array}{l}100 \mathrm{~m} \\
\text { Sens } \\
\text { Spec } \\
\text { Accur }\end{array}$ & $\begin{array}{r}+ \text { Pulm } \\
96.3 \\
92.0 \\
94.2\end{array}$ & $\begin{array}{l}100 \\
96.0 \\
98.1\end{array}$ & $\begin{array}{l}100 \\
100 \\
100\end{array}$ & $\begin{array}{l}100 \\
100 \\
100\end{array}$ & $\begin{array}{l}100 \\
100 \\
100\end{array}$ & $\begin{array}{c}100 \\
96.0 \\
98.1\end{array}$ \\
\hline $\begin{array}{l}50 \mathrm{mg} \\
\text { Sens } \\
\text { Spec } \\
\text { Accur }\end{array}$ & $\begin{array}{r}\text { Pulmo } \\
91.3 \\
94.9 \\
92.2\end{array}$ & $\begin{array}{l}95.6 \\
97.4 \\
96.5\end{array}$ & $\begin{array}{l}100 \\
100 \\
100\end{array}$ & $\begin{array}{l}100 \\
100 \\
100\end{array}$ & $\begin{array}{c}100 \\
97.4 \\
98.8\end{array}$ & $\begin{array}{c}97.8 \\
100 \\
98.8\end{array}$ \\
\hline $\begin{array}{l}50 \mathrm{mg} \\
\text { Sens } \\
\text { Spec } \\
\text { Accur }\end{array}$ & $\begin{array}{c}\text { Polyc } \\
94.4 \\
100 \\
97.0 \\
\end{array}$ & $\begin{array}{c}89.9 \\
100 \\
93.9\end{array}$ & $\begin{array}{c}83.3 \\
100 \\
90.9\end{array}$ & $\begin{array}{c}88.9 \\
100 \\
93.9 \\
\end{array}$ & $\begin{array}{c}88.9 \\
100 \\
93.9 \\
\end{array}$ & $\begin{array}{c}88.9 \\
100 \\
93.9 \\
\end{array}$ \\
\hline
\end{tabular}

Conclusions. The ${ }^{13} \mathrm{C}$-UBT is a simple, non-invasive test that can be used as the "gold standard" for the detection of H. pylori infection. Administering $50 \mathrm{mg}$ of ${ }^{13} \mathrm{C}$-Urea, a fatty test meal and a single breath sample at T30 makes it ideal even in children.

\section{A:07 COST-IMPACT OF CLARITHROMYCIN PLUS OMEPRAZOLE COMPARED TO TRADITIONAL THERAPIES FOR TREATMENT OF H. PYLORI ASSOCIATED DUODENAL ULCERS}

A. Sonnenberg. VA Medical Center, Albuquerque, NM; The Gastrointestinal Utilization Trial Study Group

Introduction: The NIH Consensus Development Conference recommended a comprehensive economic analysis of the impact of treating or not treating $H$. pylori (HP) associated ulcers. Patients were enrolled in a multicenter $(n=132)$, controlled clinical trial to determine cost savings of eradicating HP with clarithromycin plus omeprazole $(C+O)$ versus conventional anti-ulcer therapy (omeprazole $(\mathrm{O})$ or ranitidine $(\mathrm{R})$ alone).

Methods: Adult patients with HP and active duodenal ulcer were randomized to double-blinded treatment (Rx): 1) C $500 \mathrm{mg}$ TID + $040 \mathrm{mg}$
QD for 14 days followed by $O 20 \mathrm{mg}$ QD for 14 days; 2) $\mathrm{O} 20 \mathrm{mg}$ QD for 28 days; or 3) $\mathrm{R} 150 \mathrm{mg}$ BID for 28 days. Visits were performed at pre-Rx (EGD + biopsy), post-Rx (safety), and 4-6 weeks post-Rx (eradication). After the third protocol directed visit, investigators followed patients for one year by monthly telephone calls for assessment of ulcer symptoms and collection of economic data. Additional management was to be "standard of care" for that investigational site. Results: Of the 819 patients enrolled, 750 patients were eligible (confirmed ulcer and HP infection) for economic analysis. The demographics of the three groups were similar. Analysis of health resource utilization is given in the table below:

\begin{tabular}{lccc}
\hline $\begin{array}{l}\text { Resource Utilization } \\
\text { (beyond protocol) }\end{array}$ & $\begin{array}{l}\mathrm{C}+\mathrm{O} \\
(\mathrm{n}=253)\end{array}$ & $\begin{array}{l}\mathrm{O} \\
(\mathrm{n}=255)\end{array}$ & $\begin{array}{c}\mathrm{R} \\
(\mathrm{n}=242)\end{array}$ \\
\hline EGDs & 31 & 76 & 69 \\
Ulcer related clinic visits & 84 & 136 & 161 \\
Ulcer days lost from work & 116 & 122 & 787 \\
All hospitalizations & 26 & 47 & 42 \\
Ulcer related hospitalizations & 0 & 5 & 6 \\
All hospital days & 158 & 318 & 205 \\
Ulcer hospital days & 0 & 24 & 37 \\
\hline
\end{tabular}

While costs varied among the different centers, eradicating HP clearly led to a decrease in health care utilization.

Conclusion: Ours is the first prospective study to show that using antibiotics to eradicate $H$. pylori in patients with duodenal ulcer results in decreased utilization of health care resources, overall and ulcer related, when compared to conventional anti-ulcer therapy with omeprazole or ranitidine.

\section{A:25 HELICOBACTER PYLORI AND NSAIDS IN BLEEDING GASTRIC ULCER}

T.M. Ng, K.M. Fock, J.L. Khor, E.K. Teo, S.C. Chia. Division of Gastroenterology, Department of Medicine, Toa Payoh Hospital, Singapore

Helicobacter pylori ( $\mathrm{Hp}$ ) infection and Nsaids are independent risk factors for gastric ulcer (GU) and their effect may be additive. However the question as to whether they interact to influence the risk of bleeding in GU has little been studied.

We aim to determine the prevalence of $\mathrm{Hp}$ infection and Nsaids ingestion in a group of patients with GUs only and explore their roles in bleeding and nonbleeding GUs.

From January 1993 to June 1995, a total of 145 GU patients (103 M:42 $\mathrm{F}$, median age 61, range 26-94) were recruited into the study. One hundred and twenty GU patients $(83 \%)$ were $\mathrm{Hp}$ positive and $25(17 \%)$ were $\mathrm{Hp}$ negative. Nsaids ingestion within 4 weeks prior to endoscopy were present in $30 \%$, more in $\mathrm{Hp}$ negative than $\mathrm{Hp}$ positive patients $(56 \%$ vs $24 \%$ ) (p = 0.003 ). Aspirin accounted for $37 \%$ of the Nsaids consumed. Seventy-one patients have bleeding GU (49 M:22 F, median age 69, range 26-94) and 74 with nonbleeding GU (54 M:20 F, median age 58, range 29-86). Logistic regression analysis (SAS) was used to evaluate the effect of Hp infection, Nsaids ingestion, age, gender, smoking, alcohol consumption and past PUD on bleeding GU. Nsaids ingestion was the only significant risk factor found $(\mathrm{p}<0001)$. The risk of bleeding was $63.7 \%(95 \% \mathrm{CI}=55.9 \%-71.6 \%)$ if Nsaids alone was present. The probability of bleeding with $\mathrm{Hp}$ alone was $44 \%(95 \% \mathrm{CI}=35.9 \%-52.1 \%)$. When both $\mathrm{Hp}$ and Nsaid were present the risk of bleeding was $71.8 \%(95 \% \mathrm{CI}=64.6 \%-79.1 \%)$. The interaction of $\mathrm{Hp}$ and Nsaid use was statistically significant $(\mathrm{p}=0.025)$.

In conclusion, the prevalence of $\mathrm{Hp}$ infection and Nsaids ingestion in GU patients was $83 \%$ and $30 \%$ respectively. Nsaids ingestion increases the risk of bleeding in $\mathrm{GU}$ patients whereas $\mathrm{Hp}$ alone is not a risk factor. There may be some synergisms between $\mathrm{Hp}$ and Nsaids in increasing the risk of bleeding.

\section{B:27 ABERRANT EXPRESSION OF GLAND-TYPE GASTRIC MUCIN IN THE SURFACE EPITHELIUM OF H. PYLORI-INFECTED PATIENTS}

J.C. Byrd, P. Yan, C. Yunker, H. Schoeppner, R.S. Bresalier. Henry Ford Health Sci. Ctr., Detroit, MI, USA

Mucins are high- $\mathrm{M}_{\mathrm{r}}$ glycoproteins that protect the gastric epithelium. Three mucin genes, MUC6, MUC5, and MUC1, are expressed in normal stomach. Our aim was to compare expression of gastric mucin genes in HP+ patients vs uninfected (HP-neg) individuals. Gastric biopsies obtained at routine endoscopy (29 HP+ and $33 \mathrm{HP}-$ neg by histology and CLO) were examined by immunohistochemistry for mucin gene expression in surface mucous cells and mucous glands:

In HP-neg specimens, MUC6 was expressed only in mucous glands, but in $21 / 29 \mathrm{HP}+$ specimens, there was also staining of surface mucous cells. Expression of MUC5 mucin (surface but not glands) and 
$\%$ of patients with staining of surface mucous cells

\begin{tabular}{lcclcl}
\hline & HP- & HP+ & & HP- & HP+ \\
\hline MUC6 & 0 & 72 & LeX & 9 & 57 \\
MUC5 & 89 & 100 & P-ConA & 11 & 70 \\
MUC1 & 100 & 100 & Le $^{\text {b }}$ & 60 & 96 \\
\hline
\end{tabular}

MUC1 (surface and glands) was unaffected by HP infection. Carbohydrates recognized by anti-LeX (Gal $\beta 4[\mathrm{Fuc} \alpha 3] \mathrm{GlcNAc})$ and by ConA (after $\mathrm{IO}_{4} / \mathrm{NaBH}_{4}$ ) are normally found only in mucous glands, but were present in surface mucous cells in 16/28 and 16/23 of HP+ tissues. Surface $\mathrm{Le}^{\mathrm{b}}$ (Fuc $\alpha 2 \mathrm{Gal} \beta 3$ [Fuc $\alpha$ 4]GlcNAc) was present in 26/27 HP+ and 18/30 HP-neg tissues, consistent with a role for Le $e^{b}$ in HP colonization. Mucins purified from gastric aspirates of $5 \mathrm{HP}+$ patients bound more anti-MUC6 ( $0.32 \pm 0.04$ vs $0.09 \pm 0.07$ A 405/25 ng, by ELISA) and anti-Le ${ }^{b}$ (1.31 \pm 0.30 vs $0.05 \pm 0.03$ ), than mucins from $5 \mathrm{HP}$-neg patients, suggesting erosion of the altered surface mucin layer.

Conclusion: In HP+ patients, the type of mucin that is normally limited to mucous glands is also expressed in surface mucous cells. Aberrant expression of MUC6 might disrupt the protective surface mucin layer, which could be important in the pathogenesis of HP-associated diseases.

\section{B:59 THE COMPLETE DNA SEQUENCE OF THE HELICOBACTER PYLORI GENOME}

J.-F. Tomb ${ }^{1}$, G. Sutton ${ }^{1}$, A. Glodek ${ }^{1}$, L. Zhou ${ }^{1}$, O. White ${ }^{1}$, T. Utterback ${ }^{1}$, J. Kelley ${ }^{1}$, J. Peterson ${ }^{1}$, R. Fleischmann ${ }^{1}$, C. Bult ${ }^{1}$, M. Adams ${ }^{1}$, J. Gocayne ${ }^{1}$, R. Clayton ' , N. Akopyants ${ }^{2}$, D.E. Berg ${ }^{2}$, H.O. Smith ${ }^{3}$, C. Fraser ${ }^{1}$, J.C. Venter ${ }^{1} .{ }^{1}$ The Institute For Genomic Research, Rockville, MD, USA ${ }^{2}$ Washington University Medical School, St. Louis, MO, USA ${ }^{3}$ Johns Hopkins University, School of Medicine, Baltimore, MD, USA

The DNA sequence of a bacterial genome can be of immense value, especially in cases of important pathogens such as Helicobacter pylori. Here we present the complete annotated sequence of a representative $H$. pylori strain (KE26695). This strain was chosen for four reasons: (i) it colonizes piglets and elicits immune and inflammatory responses; (ii) it is of the toxigenic and Cag+ (putatively more virulent) type; (iii) its $\approx$ $40 \mathrm{~kb}$ cag region is not interrupted by rearrangement, unlike certain other Cag+ strains; (iv) it is transformable, and thus amenable to mutational tests of gene function. The genome sequence was determined using a random library of small insert plasmid clones, essentially as in our sequencing of the Haemophilus influenzae and the Mycoplasma genitalium genomes (Science 269, 496-512, 1995; Science 270, 397-403, 1995). In the initial random phase, we achieved 7.5 fold coverage by generating 24028 sequences with an average read length of 551 bases. We also sequenced the ends of 500 large fragment lambda phage clones to create a scaffold that helped ensure correct assembly. The availability of this sequence should greatly speed many areas of $\mathrm{H}$. pylori research, including characterization of colonization mechanisms, virulence traits and host interactions; analyses of $H$. pylori population structure and genome evolution; and development of new potent therapies against $H$. pylori and associated gastroduodenal diseases.
4B:06 SERO-CONVERSION AND SERO-REVERSION IN IgG ANTIBODIES TO H. PYLORI: AN 11-YEAR FOLLOW-UP OF 2,523 RANDOMLY SELECTED DANES

S.J. Rosenstock ${ }^{1,2}$, L.P. Andersen ${ }^{1}$, O. Bonnevie ${ }^{1}$, T. Jørgensen ${ }^{1,2} .^{1}$ The Copenhagen Hospital Corporation, Denmark; ${ }^{2}$ The Glostrup Population Studies, University of Copenhagen, Denmark

Purpose: To assess the 11-year sero-conversion and sero-reversion rate in IgG antibodies to $H$. pylori in adults. Methods: In 1983, 3,589 Danes aged 30-60 years entered a population-based cohort study. After 11 years, 2,656 participants attended a follow-up examination. A total of 2,523 matching pairs of sera were eligible for this study. IgG antibodies against $H$. pylori were measured with an in-house ELISA assay. Cut-off points for seronegativity and seropositivity were set at $\leq 100$ ELISA units (Eu) and $\geq 400 \mathrm{Eu}$, respectively. Participants who were IgG seronegative or IgG border-line at study entry and IgG seropositive at follow-up were regarded as sero-converters. All sero-converters had at least a four-fold increase in base-line IgG antibody levels between study entry and followup. Sero-reverters were those who were IgG seropositive at study entry and showed at least a four-fold decrease in IgG antibody levels at followup. Results: Seropositivity for IgG antibodies to $H$. pylori was seen in $24.7 \%$ [23.0-26.4] in 1983 and 24.5\% [22.8-26.2] in 1994. A total of 36 participants sero-converted within the observation period. The cumulated 11-year sero-conversion rate (incidence of $H$. pylori infection) was 18.9 [12.8-25.0] per 1,000 persons at risk. Sero-reversion was seen in $42 \mathrm{~m} / \mathrm{f}$ : $17 / 25)$ participants. The cumulated 11-year sero-reversion rate was 67.4 [47.7-87.1] per 1,000 persons at risk. Conclusions: The incidence of $H$. pylori infection is low in adults. Elimination of $H$. pylori infection seems to outbalance acquisition of the infection in adults in developed parts of the world. 